\title{
MS25-P02 | DATA PROCESSING OF 3D PRECESSION ELECTRON DIFFRACTION DATA
}

Palatinus, Lukas (Institute of Physics of the Czech Academy of Sciences, Prague, CZE); Brázda, Petr (Institute of Physics of the CAS, Prague 8, CZE)

3D electron diffraction (3D ED) has become popular recently as novel, powerful methods have been developed for structure solution and structure refinement from such data. A number of protocols have been devised for data collection. One of them is the combination of step-wise data collection with precession electron diffraction technique (PED). This technique has a number of advantages. It provides intensities well suitable for kinematical structure refinement and it is so far the only method providing data suitable for dynamical refinement. While data collected without PED can be processed by many programs including those developed for X-ray diffraction data, PED data have specific features that require special treatment.

The main specific feature of 3D ED with PED is the unique rocking curve of the reflections, which has two maxima and resembles the back of a camel. The shape of the rocking curve can be estimated by analyzing the data and determining the crystal mosaicity. Once the shape is known, the reflection intensity can be accurately extracted. Moreover, the orientation of each frame can be determined with accuracy better than $0.1^{\circ}$, allowing for the correction of possible crystal rotation during the data collection and consequently allowing for a better determination of lattice parameters and better intensity extraction.

The specifics of processing of PED data will be described and its benefits will be illustrated on several examples ranging from accurate determination of lattice parameters, through the structure solution of an extremely beamsensitive material to the determination of absolute configuration of chiral molecules. 